\title{
Oclusão Percutânea da Veia Cava Superior Esquerda Persistente Conectada Anomalamente no Teto do Átrio Esquerdo
}

\author{
Daniel Guilherme Arnoni', Jean Marcelo Ferreira da Silva', Valmir Fernandes Fontes', \\ Roberto Reyes $\mathrm{O}^{\prime}$ Connor ${ }^{1}$, Carlos Augusto Cardoso Pedra ${ }^{1}$
}

\section{RESUMO}

Relatamos um caso raro de veia cava superior esquerda persistente conectada anomalamente no teto do átrio esquerdo associada a pequena comunicação interatrial do tipo seio venoso, em criança cianótica de 9 anos, com história pregressa de abscesso cerebral. O vaso anômalo foi ocluído com sucesso no laboratório de cateterismo, utilizando-se uma prótese Amplatzer.

DESCRITORES: Cardiopatias congênitas. Cateterismo. Defeitos do septo interatrial. Veia cava superior/anormalidades. Próteses e implantes.
A persistência da veia cava superior esquerda (VCSE) drenando no seio coronário ocorre em cerca de $4 \%$ dos pacientes com cardiopatia congênita e em $0,3 \%$ da população geral ${ }^{1}$. Tal anatomia pode associar-se a comunicação interatrial $(\mathrm{CIA})$ do tipo seio coronário, com o fluxo venoso desaguando diretamente no átrio esquerdo em decorrência de deficiência parcial ou total do teto do seio coronário ${ }^{2,3}$.

Neste artigo, descrevemos um caso de conexão anômala de VCSE no teto do átrio esquerdo, associada a pequena CIA do tipo seio coronário tratado pela cardiologia intervencionista com prótese Amplatzer.

\section{RELATO DO CASO}

Criança de 9 anos, sexo masculino, pesando $21 \mathrm{~kg}$, foi encaminhada para nosso Serviço para avaliação de

1 Instituto Dante Pazzanese de Cardiologia - São Paulo, SP, Brasil. Correspondência: Carlos Augusto Cardoso Pedra. Instituto Dante Pazzanese de Cardiologia. Seção Médica de Intervenções em Cardiopatias Congênitas. Av. Dr. Dante Pazzanese, 500 - 14ํandar - São Paulo, SP, Brasil - CEP 04012-180

E-mail: cacpedra@uol.com.br

Recebido em: 13/3/2008 • Aceito em: 13/1/2009

\section{ABSTRACT}

\section{Percutaneous Occlusion of the Left Superior Vena Cava with an Anomalous Connection to the Left Atrium Roof}

A rare case of persistent left superior vena cava anomalously connected to the roof of the left atrium associated with a small coronary sinus type atrial septal defect in a cyanotic 9-year-old child with a previous history of cerebral abscess is reported. The anomalous vessel was successfully occluded in the catheterization laboratory using an Amplatzer device.

DESCRIPTORS: Heart defects, congenital. Catheterization. Heart septal defects, atrial. Vena cava, superior/abnormalities. Prostheses and implants.

doença cardíaca congênita. Apresentava história de dispneia aos médios esforços, cianose, déficit ponderal e abscesso cerebral drenado aos 7 anos. O exame físico revelou cianose (saturação em ar ambiente de 89\%), baqueteamento digital, unhas em vidro de relógio e pectus excavatum. À ausculta cardíaca, o ritmo era regular, não havia sopros e a segunda bulha apresentava normofonese com desdobramento amplo, mas não fixo. A propedêutica pulmonar e abdominal encontrava-se sem anormalidades. O eletrocardiograma revelou ritmo sinusal, com eixo a 60 graus, sem sinais de sobrecarga atrial ou ventricular e alterações da repolarização. A radiografia de tórax mostrou situs solitus, levocardia, ponta para a esquerda, área cardíaca normal e campos pulmonares sem anormalidades, além de discreto alargamento da porção esquerda do mediastino. Ecocardiogramas transtorácico e transesofágico foram realizados, evidenciando pequena $\mathrm{CIA}$ (3 $\mathrm{mm}$ a $4 \mathrm{~mm}$ ) em porções inferiores do septo interatrial com shunt da esquerda para a direita (Figura 1) e presença de grande quantidade de microbolhas de aparecimento precoce nas cavidades esquerdas. Não ficava claro o local de chegada das microbolhas. O ventrículo direito apresentava dimensões normais.

Foi indicado cateterismo cardíaco, para elucidação diagnóstica do local do shunt direita-esquerda. Sob 
Arnoni DG, et al. Oclusão Percutânea da Veia Cava Superior Esquerda Persistente Conectada Anomalamente no Teto do Átrio Esquerdo. Rev Bras Cardiol Invas. 2009;17(1):133-6.
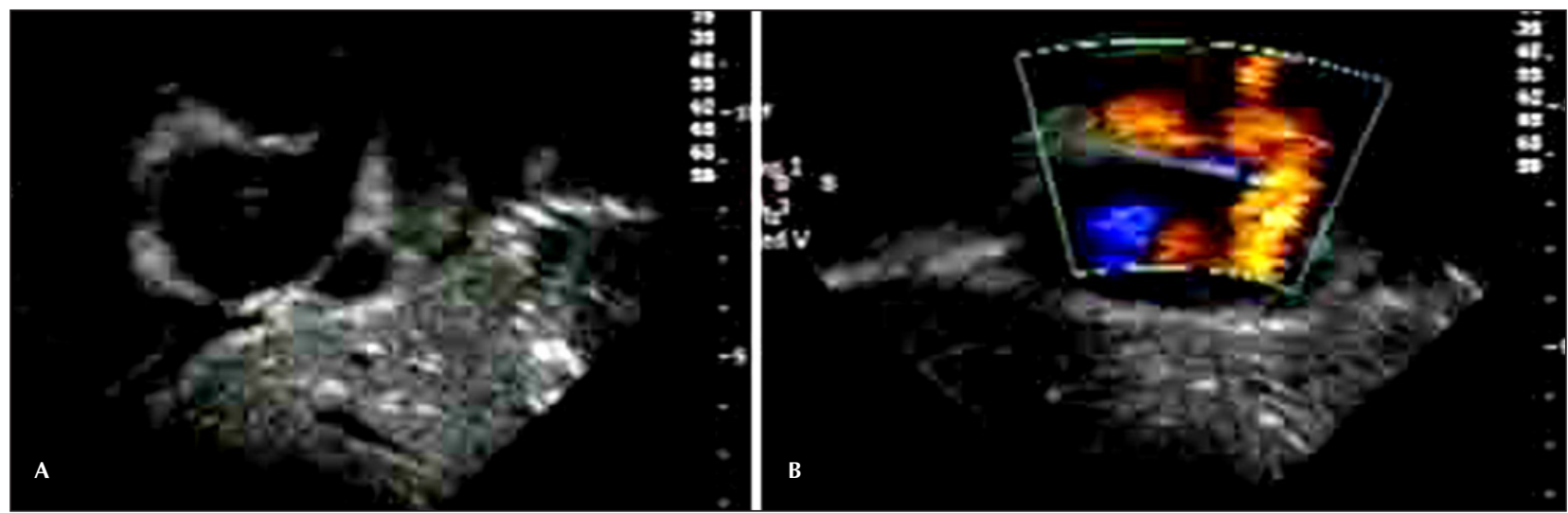

Figura 1 - Em A, imagem do ecocardiograma evidenciando comunicação interatrial do tipo seio coronário. Em B, imagem ecocardiográfica mostrando o shunt esquerda-direita pela comunicação interatrial do tipo seio coronário.

anestesia geral, realizou-se punção da veia femoral direita e foi posicionado introdutor 7 Fr. Administrou-se heparina (100 Ul/kg) e cefuroxina $(75 \mathrm{mg} / \mathrm{kg})$. As medidas pressóricas (em $\mathrm{mmHg}$ ) foram: $\mathrm{ADm}=8, \mathrm{VD}=25 / 8$, $\mathrm{TP}=25 / 12 / 17, \mathrm{AEm}=8, \mathrm{VE}=90 / 8, \mathrm{AO}=90 / 60 / 70$. O estudo oximétrico demonstrou: VCS $=76 \%$, TP $=83 \%$, $\mathrm{VP}=98 \%, \mathrm{AO}=92 \%(\mathrm{Qp} / \mathrm{Qs} 1,1)$. As angiografias mostraram veia cava superior direita (VCSD) drenando normalmente no átrio direito (diâmetro de $12 \mathrm{~mm}$ ), e presença de veia inominada de calibre reduzido $(1,5 \mathrm{~mm})$ comunicando a VCSD com a VCSE persistente (VCSEp), que drenava no teto do átrio esquerdo e media $11 \mathrm{~mm}$ a $12 \mathrm{~mm}$ (Figura 2). O átrio esquerdo possuía dimensões normais. Constatou-se formação de alça venovenosa do trajeto do cateter (Figura 3). Diante desses achados, puncionou-se novamente a veia femoral direita e posicionou-se outro introdutor $7 \mathrm{Fr}$. Com um cateter Judkins de artéria coronária direita $5 \mathrm{Fr}$ atingiuse a veia inominada e a VCSEp com ajuda do fio-guia hidrofílico. Esse cateter foi deixado na porção proximal da VCSEp, com o intuito de manter controle angiográfico durante o procedimento. Com o auxílio de um cateter balonado de furo terminal (wedge pressure), cruzou-se o seio coronário, atingindo a origem da VCSEp da veia inominada. Sobre um fio-guia extrassuporte de 0,035 polegada de troca $(260 \mathrm{~cm})$ realizou-se a troca do cateter por uma bainha longa 9 Fr (Cook).

Optou-se pela utilização de uma prótese Amplatzer de comunicação interventricular (CIV) muscular 14 mm para oclusão da VCSEp, em sua região próxima à veia inominada. O dispositivo, após carregamento clássico, foi posicionado dentro do vaso com movimentos simultâneos de empurrar o cabo liberador e puxar a bainha longa.

O controle angiográfico pelo cateter Judkins posicionado na origem da VCSEp pela veia inominada revelou posicionamento adequado da prótese e oclusão total da VCSEp. Houve aumento imediato da saturação sistêmica de oxigênio para 99\% ( $\mathrm{FiO}_{2}$ de $\left.21 \%\right)$. O dispositivo foi então liberado do cabo liberador e, após cinco a dez

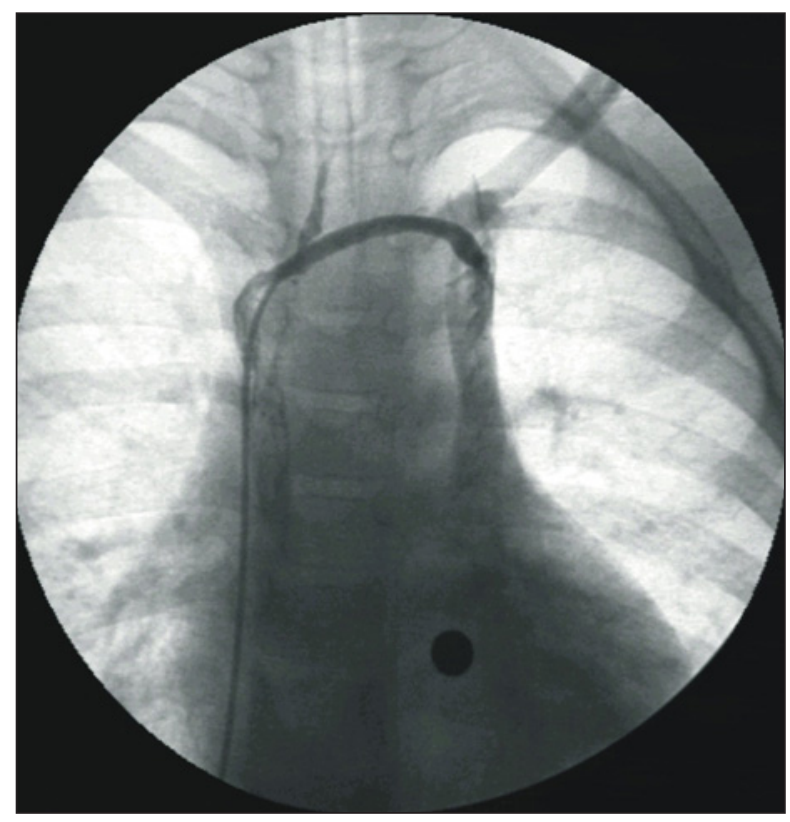

Figura 2 - Injeção de contraste na veia inominada em projeção póstero-anterior mostrando a veia cava superior esquerda drenando no teto do átrio esquerdo.

minutos, novas angiografias revelaram manutenção da posição da prótese e oclusão do vaso (Figura 4). Com isso, cateteres e introdutores foram retirados e fez-se compressão hemostática. Não houve complicações após o procedimento. A alta hospitalar foi concedida 48 horas após o procedimento, depois da realização de ecocardiograma transtorácico, que evidenciou prótese bem posicionada e ausência de microbolhas em átrio esquerdo após injeção em membro superior esquerdo.

\section{DISCUSSÃO}

Embriologicamente, a CIA do tipo seio coronário decorre de septação incompleta da veia cardinal esquerda com o átrio esquerdo, justificando a frequente 
Arnoni DG, et al. Oclusão Percutânea da Veia Cava Superior Esquerda Persistente Conectada Anomalamente no Teto do Átrio Esquerdo. Rev Bras Cardiol Invas. 2009;17(1):133-6.

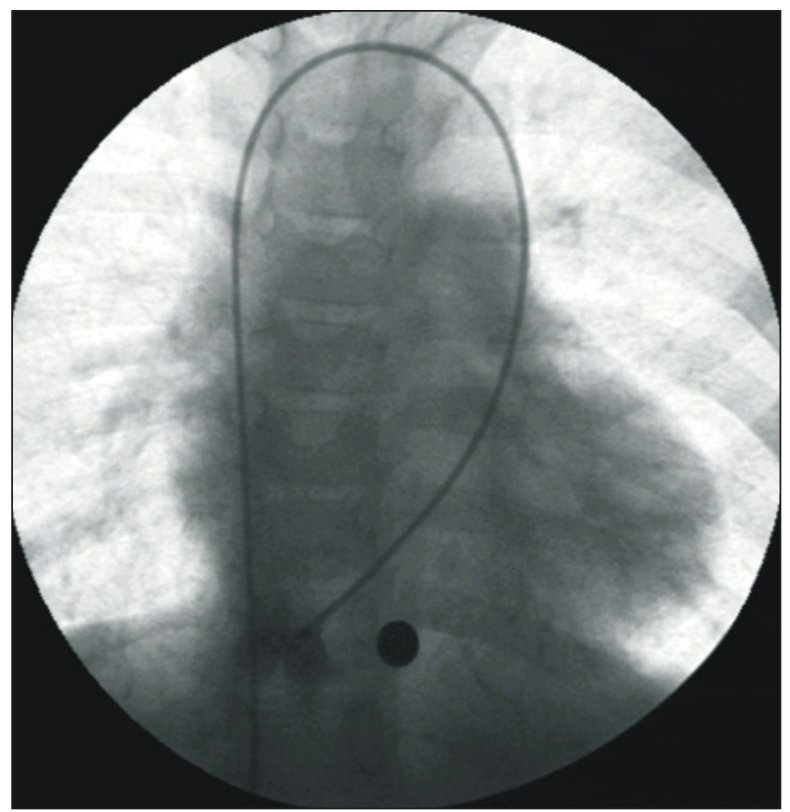

Figura 3 - Em projeção póstero-anterior, nota-se o seguinte trajeto do cateter Judkins de artéria coronária direita: veia cava inferiorátrio direito-veia cava superior direita-veia inominada-veia cava superior esquerda-átrio esquerdo-comunicação interatrial do tipo seio coronário-átrio direito. A injeção de contraste mostra a entrada da veia cava inferior no átrio direito.

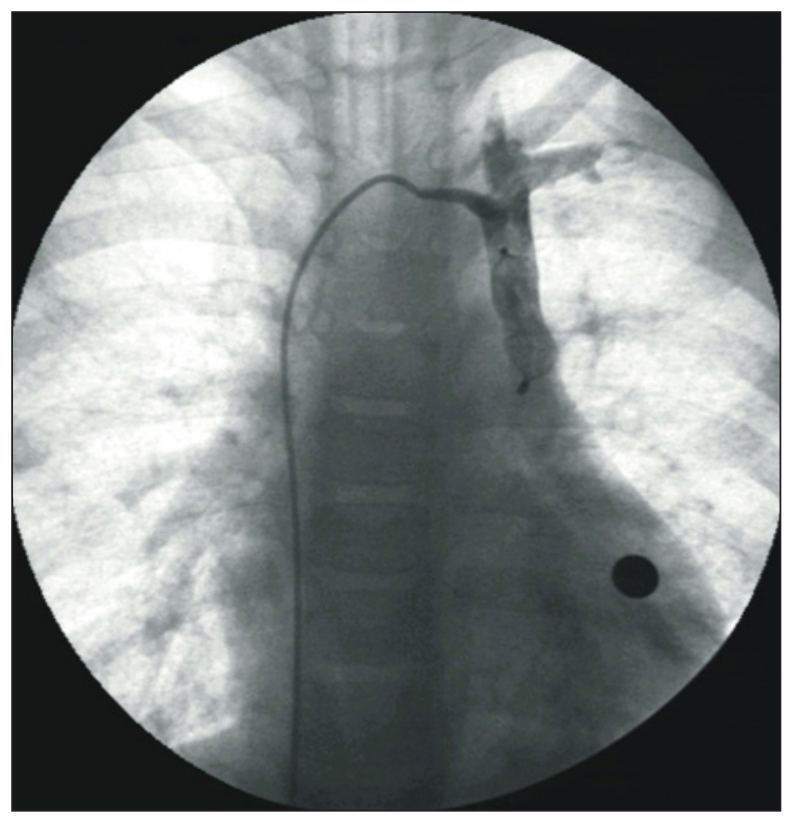

Figura 4 - Injeção de contraste na veia inominada após o implante mostrando prótese bem posicionada e veia cava superior esquerda ocluída.

associação entre VCSEp e esse tipo de $\mathrm{CIA}^{4}$. Em 1965, Raghib et al. ${ }^{5}$ relataram um caso em que havia persistência da VCSE drenando no átrio esquerdo e ausência total do seio coronário (complexo de Raghib). Como na quase totalidade dos casos o orifício do seio coro- nário está presente, atualmente prefere-se denominar tal lesão de forma anatômica (ausência parcial ou total do teto do seio coronário).

O caso relatado é considerado raro, pois a $\mathrm{CIA}$ do tipo seio coronário e a VCSEp estão frequentemente associadas a cardiopatias mais complexas, tais como isomerismos, drenagem anômala das veias pulmonares, atresia tricúspide, tetralogia de Fallot e defeito do septo atrioventricular, entre outras ${ }^{6,7}$.

Clinicamente era evidente a existência de fluxo da direita para a esquerda com insaturação sistêmica, baqueteamento digital, unhas em vidro de relógio e história pregressa de abscesso cerebral. O diagnóstico diferencial de "cianose" silenciosa deve englobar malformações arteriovenosas pulmonares, presença de forame oval com válvula de Eustáquio hipertrofiada e conexão anômala de uma veia sistêmica em cavidades esquerdas. Nesse caso, uma sombra muito tênue na radiografia de tórax assinalava para possibilidade diagnóstica da presença de VCSEp.

$\mathrm{Na}$ investigação inicial por meio da ecocardiografia, o diagnóstico não foi completamente elucidado. Entretanto, confirmou-se a presença de grande fluxo direitaesquerda em decorrência do aparecimento precoce de microbolhas nas cavidades esquerdas e da presença de uma pequena CIA em porções inferiores do septo interatrial. O paciente foi, então, levado ao laboratório de hemodinâmica com a hipótese de haver fístulas arteriovenosas pulmonares ou alguma conexão anômala venoatrial. A angiografia foi diagnóstica, o que possibilitou o tratamento do vaso anômalo em uma única sessão terapêutica.

Do ponto de vista do tratamento percutâneo, além da prótese de CIV muscular utilizada, poderíamos ter empregado outros dispositivos da família Amplatzer (para oclusão da CIA, do forame oval ou persistência do canal arterial $\left.{ }^{8-10}\right)$. Os plugues vasculares Amplatzer, recém-introduzidos para uso clínico, também seriam uma excelente opção, porém não estavam disponíveis no momento do procedimento ${ }^{11}$. É importante salientar o método de escolha desses tipos de dispositivos: seu disco deve ter diâmetro cerca de 50\% maior que o diâmetro da veia, para que exerça força radial considerável nas paredes internas do vaso, evitando seu deslocamento. Isso é de suma importância para vasos venosos, mais complacentes que os arteriais. Para artérias ou vasos mais rígidos, talvez um diâmetro do dispositivo 30\% maior que o diâmetro máximo do vaso seja suficiente para estabilizá-lo em uma posição satisfatória. Além disso, deve-se liberar a prótese tomando-se o cuidado de não ocluir nenhuma estrutura vital vizinha, no caso o seio coronário e a veia inominada. O superdimensionamento também deve ser evitado, para que a prótese não fique alongada, estendendo-se para fora da estrutura a ser embolizada ${ }^{12}$. No caso relatado, não houve preocupação em relação ao possível comprometimento da drenagem do lado venoso 
superior esquerdo do corpo, por causa do tamanho reduzido da veia inominada. Após a oclusão, a veia inominada geralmente se dilata, proporcionando drenagem adequada, conforme a demanda e a necessidade. Além disso, vasos colaterais se formam no pescoço e no mediastino para ajudar a drenagem, de forma semelhante ao que é observado nas cirurgias de Glenn e Fontan.

As molas de Gianturco não seriam uma boa opção nesse caso, pois não havia disponível um diâmetro ideal para o fechamento do vaso. Entretanto, esse dispositivo poderia ter sido implantado pela veia inominada se houvesse fluxo residual pela prótese Amplatzer, o que é raro nas oclusões venosas, de fluxo lento.

Finalmente, optamos por não intervir na CIA do tipo seio coronário pelo seu pequeno tamanho e pela sua repercussão hemodinâmica limitada com Qp/Qs de apenas 1,1. Com isso, o tratamento cirúrgico com sua conhecida morbidade foi evitado.

\section{CONCLUSÃO}

Este relato ilustra a exequibilidade, a segurança e a eficácia do tratamento percutâneo de um caso raro de conexão anômala da VCSEp no teto do átrio esquerdo associado a pequena $\mathrm{CIA}$ do tipo seio coronário parcialmente sem teto, utilizando-se uma prótese da família Amplatzer.

\section{CONFLITO DE INTERESSES}

Dr. Carlos A. C. Pedra é consultor da empresa AGA Medical Corporation, fabricante da prótese Amplatzer, e da empresa Bioassist, representante da AGA no Brasil. Os demais autores declararam inexistência de conflito de interesses.

\section{REFERÊNCIAS BIBLIOGRÁFICAS}

1. Freedom RM, Mawson JB, Yoo SJ, Benson LN. Anomalies of systemic venous connections, coronary sinus and divi- ded right atrium. In: Freedom RM, Mawson JB, Yoo SJ, Benson LN, editors. Congenital heart disease. Textbook of angiography. Armonk: Futura Publishing; 1997. p.291-337.

2. Adatia I, Gittenberger-de Groot AC. Unroofed coronary sinus and coronary sinus orifice atresia. Implications for management of complex congenital heart disease. J Am Coll Cardiol. 1995;25(4):948-53.

3. Freedom RM, Culham JA, Rowe RD. Left atrial to coronary sinus fenestration (partially unroofed coronary sinus). Morphological and angiocardiographic observations. Br Heart J. 1981;46(1):63-8.

4. Almeida FA, Pedra CA, Jesus CA, Pedra SR, Fontes VF, Sousa LC. Comunicação do tipo seio coronário, comunicação interventricular e ausência de veia cava superior esquerda. Arq Bras Cardiol. 1998;71(4):613-7.

5. Raghib G, Ruttenberg HD, Anderson RC, Amplatz K, Adams $P \mathrm{Jr}$, Edwards JE. Termination of left superior vena cava in left atrium, atrial septal defect, and absence of coronary sinus: a developmental complex. Circulation. 1965;31:906-18.

6. Medeiros Sobrinho JK, Fontes VF, Pontes Jr SC. Defeitos isolados do septo atrial. In: Medeiros Sobrinho JK, Fontes VF, Pontes Jr SC, editors. Cardiopatias congênitas. São Paulo: Sarvier; 1990. p.295-314.

7. Quaegebeur J, Kirklin JW, Pacifico AD, Bargeron LM Jr. Surgical experience with unroofed coronary sinus. Ann Thorac Surg. 1979;27(5):418-25.

8. Gibbs JL. Interventional catheterisation. Opening up II: venous return, the atrial septum, the arterial duct, aortopulmonary shunts and aortopulmonary collaterals. Heart. 2000; 83(2):237-40.

9. Piéchaud JF. Closing down: transcatheter closure of intracardiac defects and vessel embolisations. Heart. 2004;90(12): 1505-10.

10. Rothman A. Pediatric cardiovascular embolization therapy. Pediatr Cardiol. 1998;19(1):74-85.

11. Hill SL, Hijazi ZM, Hellenbrand WE, Cheatham JP. Evaluation of the Amplatzer vascular plug for embolization of peripheral vascular malformations associated with congenital heart disease. Catheter Cardiovasc Interv. 2006;67(1):113-9.

12. Dumit MR, Torres AG. Cierre percutáneo de fístulas coronárias. In: Sousa A, Buitrón F, Hayashi EB, Sousa JE, editors. Intervenciones cardiovasculares - SOLACI. São Paulo: Atheneu; 2005. p.493-8. 